\title{
Apparent Image Formation by Compton-Scattered Photons in Gamma-Ray Imaging
}

\author{
Mai K. Nguyen, C. Faye, L. Eglin, and T. T. Truong
}

\begin{abstract}
The solution to the scatter problem remains a major technical challenge in gamma ray imaging. This paper presents a modelization of apparent images formed by scattered photons at various energies. It may be used either to generate a series of realistic multi-energy images or be included in an image enhancement procedure.
\end{abstract}

Index Terms-Apparent images, gamma ray imaging, images indexed in energy, multi-energy image, scattering, single photon emission computed tomography (SPECT).

\section{INTRODUCTION}

G AMMA ray imaging allows the visualization of hidden structures by detection of gamma radiation distributed within the objects under study. This is used in much of imaging modalities widely found in industrial testing by nondestructive control techniques, inspection of nuclear sources, and nuclear medicine. However, the quality of gamma images is very poor in terms of spatial resolution and SNR. This is due to many degrading factors in gamma detectors such as low performance of gamma optics, Poisson noise of photon emission, and several physical processes (attenuation, photoelectric effect, and Compton scattering in biological media). In particular, Compton scattering causes deflection of photons and their energy loss. This leads to false sources, blurs in images, reduction of image contrast, and consequently to poor image quality [1]. Finding the solution to the scatter problem remains a major technical challenge [2]-[5].

Up until now, efforts have been concentrated in the elimination of scattered photons. The most common idea is to separate the scattered components from the nonscattered (primary) ones and only keep the primary events. Whatever the method used, scattered photons are either subtracted off from the overall counts or filtered with the use of narrow energy windows. But it is not clear that discarding Compton scattered photons is the appropriate solution.

In fact, a primary (direct) image of the object is obtained when $\gamma$-photons emitted from the object have gone through the medium without Compton scattering and have directly entered the collimator along the vertical axis of the camera. The number

Manuscript received December 24, 2000. The associate editor coordinating the review of this paper and approving it for publication was Prof. H. Maitre.

M. K. Nguyen, C. Faye, and L. Eglin are with the Equipe de Traitement des Images et du Signal, CNRS UPRESA 8051/ENSEA/Université de Cergy-Pontoise, Ecole Nationale Supérieure de l'Electronique et ses Applications, 95014 Cergy-Pontoise, France (e-mail: nguyen@ensea. fr).

T. T. Truong is with the Laboratoire Physique Théorique et Modélization, Université de Cergy-Pontoise, 95031 Cergy-Pontoise Cedex. France.

Publisher Item Identifier S 1070-9908(01)08830-7. of such photons is unfortunately very small compared to the total number of emitted photons by the object. This ideal image although in one-to-one correspondence with the object is quite weak and also blurred by noise, secondary scattering against the collimator walls, fluctuations of measurement electronics, etc. Thus, the quality of this image is poor and some improvement is necessary. This problem is discussed elsewhere [6].

In currently used gamma cameras, typically $70 \%$ to $80 \%$ of the incident photons are discarded by the photopeak energy window. Moreover, among those retained, roughly $30 \%$ to $40 \%$ should not have been taken because they have suffered from scattering. So this kind of separation technique leads to a net loss of photons collected and a very bad SNR. This is especially penalizing for detections of small spots and medical applications where both accuracy and low dose of radiation are required.

Although the scattered photons carry less information about the source than the primary ones, they may turn out to be useful to improve the image quality. This is particularly true when one considers the backscattered photons (i.e., photons which follow a back and forth path). From the camera point of view, these photons appear to come from the right location of the object. Taking into account these scattered photons may lead to a slight improvement in the image resolution, but more likely, to a significant reduction of noise-affecting images.

In our approach [7], instead of rejecting the scattered photons, it is proposed that we account for them in image processing from formation to restoration. The performance of an imaging system depends first on the accuracy of image formation modeling. This paper shows how take into account the scattered photons in the image formation process.

After Compton collisions, the emerging photons carry less energy than incident ones and are deflected. Thus, photons arriving at the cameras have energies that are continuously distributed below the incident energy and they do not necessarily come from the same point source. These photons build up secondary apparent images at various energies, superimposed on the direct image. The set of these images (direct and apparent), form the so-called multi-energy image sequences.

Our aim is to study the nature of secondary apparent images of an object from Compton-scattered photons at different energies. It may be relevant for constructing a new model of multi-energy image formation. The idea is particularly suited for the last generation of medical single photon emission computed tomography (SPECT) cameras operating in "list" mode since both energy and location of the photon are recorded.

We limit ourselves to single (first-order) Compton scattering. This is in fact the case for scattering events in the photopeak 
window [5]. Higher-order scattering will be considered in a subsequent work. The first-order multi-energy image sequences are created in reasonable computation time, as opposed to Monte Carlo simulations, which require excessive time and huge memory and which must be performed anew for each patient.

In this paper, Section II describes the formation of apparent images at various energies. In Section III, examples of such types of images from a thyroid phantom are presented as illustrations and their quality is commented.

\section{FORMATION OF APPARENT IMAGES}

We consider here the intensity at a detector pixel due to one point source on the object as function of the scattered photon energy. From emission to reception in camera detector, a photon may undergo several Compton collisions. In this work, we shall be concerned solely with processes involving one Compton scattering for photons.

To catch the essential of the image formation mechanism we shall consider the following simple system (Fig. 1).

The object is flat and lies in a plane perpendicular to the axis of the camera. It is limited by a simple curve $\mathcal{C}$, i.e., a curve described by a simple equation. Let $P$ be the detector pixel with coordinates $x_{P}, y_{P}, z_{P}=0$, at which Compton-scattered photons in the upper medium arrive and $S$ be a point source on the planar object. It is assumed that $S$ emits isotropically in all directions. Call $I_{0}(S)$ the number of $\gamma$-photons emitted per unit of time by a unit area of the planar object around $S$. Let $M$ be a Compton collision site in the upper medium. The number of photons arriving at $M$ per unit of time and per solid angle unit from $S$ is

$$
\frac{I_{0}(S)}{r^{2}} \exp (-\mu r)
$$

where $r=S M$ and $\mu$ is the attenuation coefficient of the upper medium.

At $M$, the photon interacts with an electron at rest, the incident photon has lost energy, it emerges from the collision with an energy $E_{1}$ in a direction making an angle $\theta_{1}$ with the incident direction as given by the Compton formula [9]

$$
E_{1}=E_{0}\left[1+\epsilon\left(1-\cos \theta_{1}\right)\right]^{-1}
$$

where $\epsilon=E_{0} / m c^{2}$ is the ratio between the incident photon energy and the rest energy of the electron.

The photon has only a finite probability for emerging in this direction and arriving at $P$, which is expressed by a differential cross section $d \sigma / d \Omega$. Thus, the total number of emerging photons per unit time in the direction of the elementary solid angle $d \Omega$ is

$$
\frac{I_{0}(S)}{r^{2}} \exp (-\mu r) \frac{d \sigma}{d \Omega} \frac{1}{z_{M}^{2}} \exp \left(-\mu\left(z_{M}-l\right)\right)
$$

where $z_{M}$ is the vertical coordinate of $M, l$, the distance from $P$ to the upper medium (see Fig. 1), and

$$
\frac{d \sigma}{d \Omega}=\frac{1}{2} r_{e}^{2} P\left(\theta_{1}\right)
$$

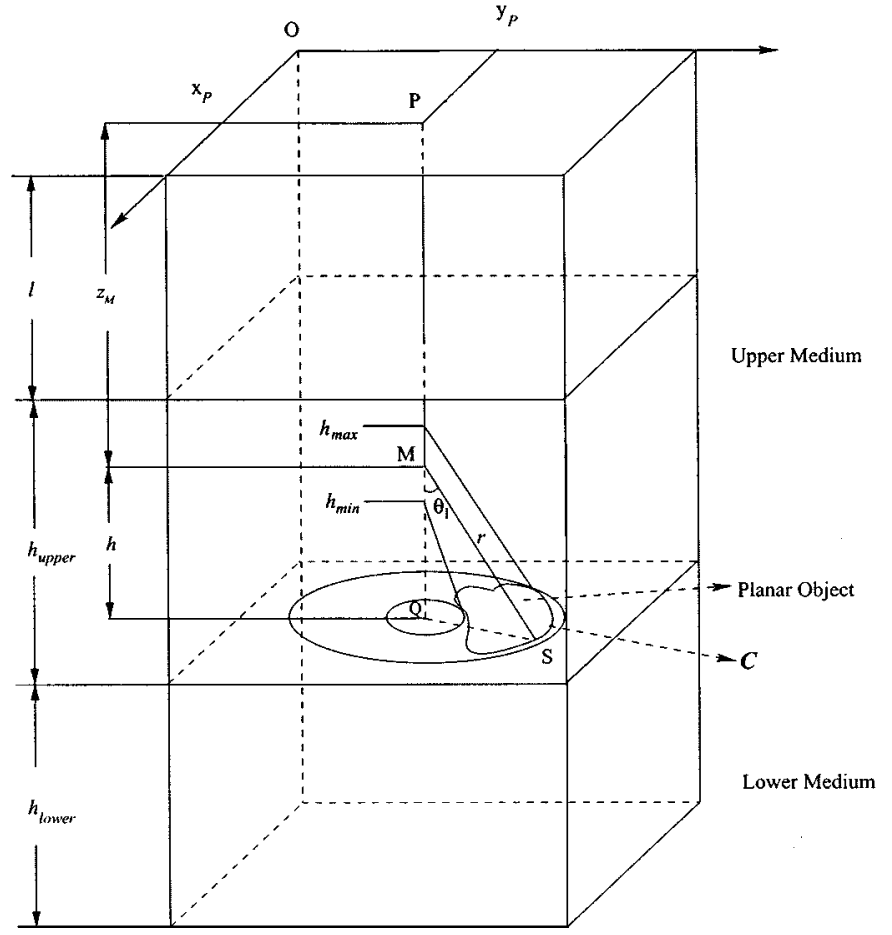

Fig. 1. Schematic arrangement of the system.

$P\left(\theta_{1}\right)$ is given by the so-called Klein-Nishina formula [9]

$$
\begin{aligned}
P\left(\theta_{1}\right)=\left(1+\epsilon\left(1-\cos \theta_{1}\right)\right)^{-2} & \\
& \times\left[1+\cos ^{2} \theta_{1}+\frac{\epsilon^{2}\left(1-\cos \theta_{1}\right)^{2}}{1+\epsilon\left(1-\cos \theta_{1}\right)}\right] .
\end{aligned}
$$

Here, $r_{e}$ is the "radius" of the electron $r_{e}=2.818 \times 10^{-15} \mathrm{~m}$. It is interesting to note that we start out with primary isotropic sources but secondary sources are completely anisotropic.

Now the total number of photons reaching the collimator due to point sources in an elementary area of the object $\rho d \rho d \phi$, where $\rho$ and $\phi$ are local polar coordinates of $S$, as in Fig. 1, is $d^{2} \mathcal{I}\left(\theta_{1}\right)$

$d^{2} \mathcal{I}\left(\theta_{1}\right)=n_{e} \frac{I_{0}(S)}{r^{2}} \exp (-\mu r) \frac{d \sigma}{d \Omega} \frac{1}{z_{M}^{2}} \exp \left(-\mu\left(z_{M}-l\right)\right) \rho d \rho d \phi$

with $n_{e}$ the number of free electrons per unit volume of upper medium.

If $Q$ is the projection of $P$ onto the object plane, then we call $M Q=h$, (see Fig. 1) $d^{2} \mathcal{I}\left(\theta_{1}\right)$ has now the expression

$$
\begin{aligned}
& d^{2} \mathcal{I}\left(\theta_{1}\right)=\frac{n_{e} r_{e}^{2}}{2} I_{0}(S) \\
& \times\left[P\left(\theta_{1}\right) \sin ^{2} \theta_{1} \exp \left(-\frac{h \mu}{\cos \theta_{1}}\right)\right] F\left(z_{M}\right) \frac{d h}{h} d \phi
\end{aligned}
$$

where $F\left(z_{M}\right)=\left(1 / z_{M}^{2}\right) \exp \left(-\mu\left(z_{M}-l\right)\right)$.

The quantity $\left[P\left(\theta_{1}\right) \sin ^{2} \theta_{1} \exp \left(-h \mu / \cos \theta_{1}\right)\right]$ is the dimensionless part of $d^{2} \mathcal{I}\left(\theta_{1}\right)$. It varies as a function of $\theta_{1}$ or $E_{1} / E_{0}$ due to first-order Compton effect. Its theoretical curve goes to zero at photopeak energy $E_{0}$ and at backscattered photon energy $E_{0}(1+2 \epsilon)^{-1}$ exactly and the form of this curve is reminiscent of the one found by Monte Carlo method in the literature [8]. 
Given the line $P Q$ joining the detector pixel to its projection onto the object plane, the collision site $M$ may be anywhere on this line in the upper medium, i.e., $h$ belongs to the range $0<h \leq h_{\text {upper. }}$. The case $h=0$ is to be excluded since for $h=0$, a photon emitted at site $Q$ must come out perpendicularly to the object plane and therefore cannot be Compton scattered. This photon having energy $E_{0}$ belongs to the direct image and must not be counted in the formation of the apparent image. This requirement can be achieved by declaring that $I_{0}(S)$ be identically zero for $S=Q\left(I_{0}(S)=0\right)$.

To get the total intensity at $P$ per time unit, we must integrate over $h$ and $\phi$ since we collect contributions of all point sources on the object. For this, it necessary to determine the integration ranges.

First, we fix $h$ in the semi-open interval $] 0, h_{\text {upper }}$. This determines a collision site $M$ on $P Q$. The cone of apex $M$ and opening angle $\theta_{1}$ intersects the planar object along an arc of circle (of radius $\rho=h \tan \theta_{1}$ ) limited by two ends with angular coordinates $\phi_{\text {begin }}$ and $\phi_{\text {end }}$. Any point source $S$ on this arc could emit a photon that Compton scatters at $M$ and winds up at $P$. Expressing the coordinates of $S$ in the reference of the collimator as $x_{S}=x_{P}+h \tan \theta_{1} \cos \phi, y_{S}=y_{P}+h \tan \theta_{1} \sin \phi$, and $z_{P}=l+h_{\text {upper }}$, we see that the $\phi$-integration is merely a mean value on an arc of circle

$$
\left\langle I_{0}\left(P, h, \theta_{1}\right)\right\rangle=\int_{\phi_{\mathrm{bcgin}}}^{\phi_{\mathrm{cnd}}} I_{0}\left(x_{S}, y_{S}, l+h_{\text {upper }}\right) d \phi .
$$

Now the limits of the $h$-integration depend essentially on the shape of the planar boundary $\mathcal{C}$ of the object. In general, for given $\theta_{1}$ (or energy $E_{1}$ ), there will be a cone of apex $M$ and opening angle $\theta_{1}$, which contains the entire object in its circular basis, the boundary of which being tangent at least at one point to $\mathcal{C}$. The height of this cone belongs to the interval $\left.] 0, h_{\text {upper] }}\right]$ and will be called $h_{\max }$. If $Q$ (the projection of $P$ onto the object plane) lies inside the object, clearly the lower limit of the $h$-integration is zero, with the restriction on $I_{0}(S)$ explained previously. But if $Q$ does not belong to the object (this is the case when $\mathcal{C}$ is of the shape of a horse-shoe surrounding $Q$ ), there will be a smallest cone of height $h_{\min }$ and opening angle $\theta_{1}$, which does not contain any part of the object and is tangent to $\mathcal{C}$ at at least one point. Consequently, the total intensity per time unit received at $P$ due to the object for $0<\theta_{1}<\pi / 2$ is

$$
\mathcal{I}\left(x_{P}, y_{P} ; E_{1}\right)=\frac{n_{e} r_{e}^{2}}{2}\left[P\left(\theta_{1}\right) \sin ^{2} \theta_{1}\right] \mathcal{K}\left(P, E_{1}, \mathcal{C}\right)
$$

with $\mathcal{K}\left(P, E_{1}, \mathcal{C}\right)$ being the integral

$$
\int_{h_{\min }}^{h_{\max }} F\left(z_{M}\right) \exp \left(-\frac{h \mu}{\cos \theta_{1}}\right)\left\langle I_{0}\left(P, h, \theta_{1}\right)\right\rangle \frac{d h}{h} .
$$

Next we examine the dependence of $\mathcal{I}\left(x_{P}, y_{P} ; E_{1}\right)$ on $E_{1}$ in the energy range $\left[E_{0}(1+\epsilon)^{-1}, E_{0}[\right.$. Near the photopeak energy $E_{0}$, the image is primarily a direct image with almost no contribution of Compton-scattered photons, this image should suffer no blurring. But for energies near $E_{0}(1+\epsilon)^{-1}$, the deflection angle $\theta_{1}$ is near $\pi / 2$ and will contribute only scattering sites $M$ near $Q$. But then $S$ should be far away, hence, beyond the

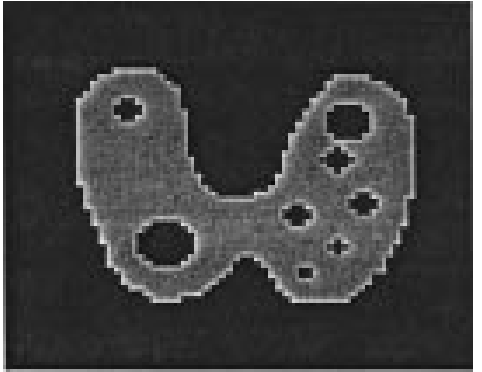

Fig. 2. Thyroid phantom containing cold nodules.

boundary $\mathcal{C}$ of the object, where $I_{0}(S)$ is zero. Thus, one expects that for $\theta_{1} \sim \pi / 2$, the image will be of very bad quality.

Given $\theta_{1}$ or $E_{1}$ and the (vertical) direction of the outgoing photon from a scattering site $M$ in the upper medium, all possible incident photons from the object must lie on a cone of apex $M$ and opening angle $\theta_{1}$. It seem that the photon scattered at the energy $E_{1}$ come from a virtual object included in the volume of scattering medium. This virtual object is characterized by its apparent activity which is linked to the activity of the original object by the relation (9).

When varying the energy $E_{1}$ in the interval $\left[0, E_{0}\right.$ [ a set of apparent images is obtained. It forms with the direct image a sequence of images indexed in energy (the so-called the multienergy image series).

In our simulations, the Technetium ${ }^{99 m} \mathrm{~T}_{c}$ is employed, with $E_{0}=140 \mathrm{keV}$. Concerning the collimator and detector modeling, we used a standard model of collimator (with parallel holes), whose response has a simple conical form with zero value for incidence angles greater than the collimator aperture. Next, the effects of the detector (scintillator-photomultiplier tubes-measurement electronics) are modeled by a three-dimensional (3-D) Gaussian transformation (two space dimensions and one energy dimension), which describes the errors due to the detector in evaluating positions and energy of the detected photons. Finally, we add the Poisson noise of photon emission. Combining all these additional modelizations, we obtain the final series of images indexed in energy. The result is presented in Fig. 3.

Our approach taking into account of the single scattered photons offers two advantages: it yields a realistic representation of the first-order multi-energy image formation process and reduces the computational time by a factor of 100 , as compared to the Monte Carlo method.

\section{RESULTS AND DISCUSSIONS}

Fig. 2 presents a thyroid phantom containing cold nodules (original object). In Fig. 3, we give some images out of a series of 20 images generated at each energy interval $\triangle E=$ $4 \mathrm{keV}$ starting from $E_{0}=140 \mathrm{keV}$. There is a good resemblance with actual images obtained from phantoms. The computation of these $20(64 \times 64)$ images lasted only five minutes on a mid-range workstation using MatLab. One can observe that the images at photopeak energy $E_{0}=140 \mathrm{keV}$ and at backscattered photon energy $E_{1}=90.44 \mathrm{keV}$ (for $\theta_{1}=\pi$ ) are of good quality, whereas the image at near right angle scattering $E_{0} \approx 112 \mathrm{keV}$, (for $\theta_{1} \approx \pi / 2$ ) as expected, is the most 


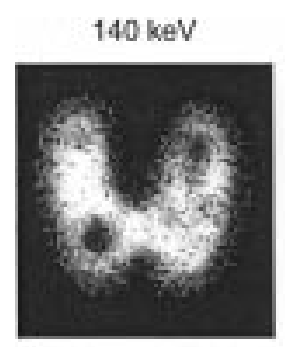

$112 \mathrm{keV}$

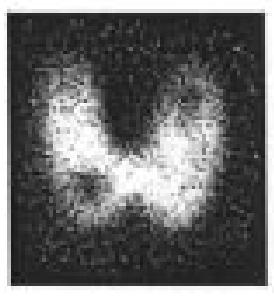

$136 \mathrm{keV}$

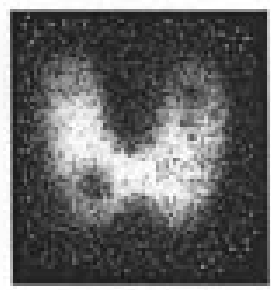

$104 \mathrm{koV}$

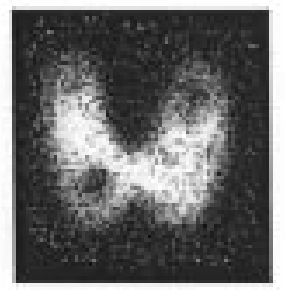

$128 \mathrm{keV}$

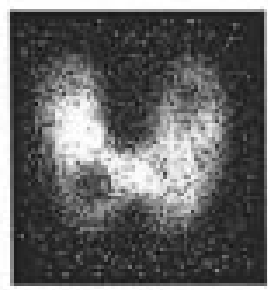

$96 \mathrm{keV}$

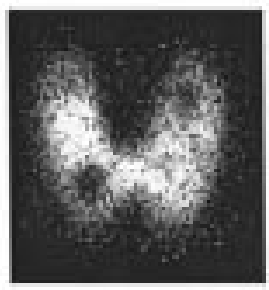

$120 \mathrm{keV}$

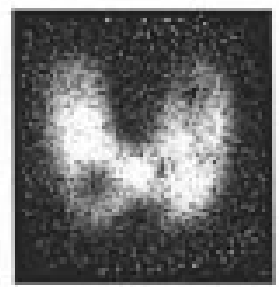

$90.44 \mathrm{keV}$

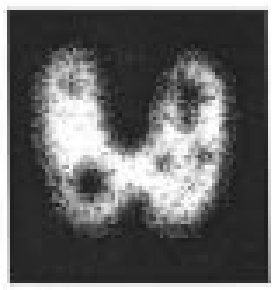

Fig. 3. Multi-energy image sequence of the thyroid phantom.

affected. The others apparent images corresponding to $E_{1}=$ $136,128,120,104,96 \mathrm{keV}$ are blurred by Compton scattering.

This idea of the multi-energy approach is found useful readily in other domains. We all know that a colored picture of an object is the superposition of many images taken at different colors or visible photon energies. Infrared images of the earth surface have revealed unexpected features compared to images taken in the visible spectrum. And images of galaxies taken with X-ray or Gamma-ray telescopes have brought extra information to regular optical telescope pictures. However in these situations one always assumes that point sources on the object emit photons in a wide range of energies.

The hope is that the apparent images produced by Comptonscattered photons once adequately modeled may be used either in the image formation process to generate a series of realistic images indexed in energy or be included into an image enhancement procedure (subject of future work). Since the computation time is reasonably short, the multi-energy approach may be of interest for medical applications. Contributions of higher-order Compton scattering will be considered in future work with the same spirit.

\section{REFERENCES}

[1] M. S. Rosenthal, J. Cullom, W. Hawkins, S. C. Moore, B. M. W. Tsui, and M. Yester, "Quantitative SPECT imaging: A review and recommendations by the focus committee of the society of nuclear medicine computer and instrumentation council," J. Nucl. Med., vol. 36, pp. 1489-1513, 1995.

[2] I. Buvat, H. Benali, A. Todd-Pokropek, and R. Di Paola, "Scatter correction in scintigraphy: The state of the art," Eur. J. Nucl. Med., vol. 21, pp. 675-694, July 1994.

[3] D. R. Haynor, R. L. Harrison, and T. K. Lewellen, "Energy-based scatter correction for 3D PET: A Monte Carlo study of best possible results," in Proc. Fully Three-Dimensional Image Reconstruction in Radiology and Nuclear Medicine, Philadelphia, PA, 1997, pp. 52-54.

[4] M. Ljungberg and S. E. Strand, "Scatter and attenuationcorrection in SPECT using density maps and Monte Carlo simulated scatter functions," J. Nucl. Med., vol. 31, pp. 1560-1567, 1990.

[5] J. M. Ollinger, "Model-based scatter correction for fully 3D PET," Phys. Med. Biol., vol. 41, pp. 153-176, 1996.

[6] M. K. Nguyen, H. Guillemin, and P. Duvaut, "Bayesian MAP restoration of scintigraphic images," in Proc. IEEE Int. Conf. Acoustics, Speech, and Signal Processing, Phoenix, AZ, 1999.

[7] C. Faye, M. K. Nguyen, and L. Eglin, "A joint energy-spatial model for formation of a gamma image sequence indexed in energy," in Proc. SPIE, vol. 3659, J. M. Boone and J. T. Dobbins, Eds., 1999, pp. 285-295.

[8] H. Zaidi, "Relevance of accurate Monte Carlo modeling in nuclear medical imaging," Med. Phys., vol. 26, no. 4, pp. 574-608, 1999.

[9] F. H. Attix, Introduction to Radiological Physics and Radiation Dosimetry. New York: Wiley, 1986. 